\title{
Nonlinear free and forced vibration of Euler- Bernoulli beams resting on intermediate flexible supports
}

\author{
Hatim Fakhreddine ${ }^{1 *}$, Ahmed Adri ${ }^{1}$, Said Rifai ${ }^{1}$ and Rhali Benamar ${ }^{2}$ \\ ${ }^{1}$ Laboratoire de Mécanique Productique et Génie Industriel, Ecole Supérieure de Technologie, Hassan II \\ University of Casablanca, B.P.8012, Oasis, Casablanca, Maroc \\ ${ }^{2}$ Mohammed V University in Rabat, EMI-Rabat, LERSIM, B.P.765 Agdal, Rabat, Morocco
}

\begin{abstract}
This paper deals with the geometrically nonlinear free and forced vibration analysis of a multi-span Euler Bernoulli beam resting on arbitrary number $\mathrm{N}$ of flexible supports, denoted as BNIFS, with general end conditions. The generality of the approach is based on use of translational and rotational springs at both ends, allowing examination of all possible combinations of classical beam end conditions, as well as elastic restraints. First, the linear case is examined to obtain the mode shapes used as trial functions in the nonlinear analysis. The beam bending vibration equation is first written in each span. Then, the continuity requirements at each elastic support are stated, in addition to the beam end conditions. This leads to a homogeneous linear system whose determinant must vanish in order to allow nontrivial solutions to be obtained. Numerical results are given to illustrate the effects of the support stiffness and locations on the natural frequencies and mode shapes of the BNIFS. The nonlinear theory is then developed, based on the Hamilton's principle and spectral analysis. The nonlinear beam transverse displacement function is defined as a linear combination of the linear modes calculated before. The problem is reduced to solution of a non-linear algebraic system using numerical or analytical methods. The nonlinear algebraic system is solved using an explicit method developed previously (second formulation) leading to the amplitude dependent nonlinear fundamental mode of the BNIFS.
\end{abstract}

\section{Introduction}

The purpose of this paper is to develop a model for the nonlinear free and forced transverse vibrations of beams supported by a finite number of flexible supports. Such a model may be applied for instance to bridges, to Earthquake-resistant buildings, to vehicles or industrial machines supported by springs, working in severe environment or subjected in the neighbourhood of resonances to geometrical nonlinearity. In order to investigate the geometrically nonlinear dynamic behaviour of Euler-Bernoulli beams, with finite number of intermediate flexible supports located at arbitrary positions, in both free and forced cases, the Hamilton's principle and spectral analysis are used, as in [1-4].First, linear case is examined to obtain the mode shapes in each span. Then, the continuity requirements at each elastic support are stated. The beam end supports are modelled by rotational and translational springs, allowing examination of all possible combination of classical beam end conditions as well as the case of elastic supports, by varying the spring stiffness. Then the transfer matrix 
method is adopted to get a reduced set of algebraic system solved by the one dimensional Newton-Raphson iteration method. Subsequently, the expressions for the kinetic energy, the total strain energy due to bending and the nonlinear strain energy due to the axial load induced by the large deflections, are developed. By applying Hamilton's principle with integration of the time functions over a period of vibration, or Lagrange's equations [1] combined with the harmonic balance method (HBM), the nonlinear amplitude equation is obtained as a set of nonlinear algebraic system solved by injecting initial estimates for the basic function contribution coefficients in a multidimensional Newton-Raphson iteration algorithm. The nonlinear mode shapes are finally obtained as the sum of the contribution coefficient multiplied by the linear mode shapes used here as basic functions in the nonlinear analysis [2-3]. In the forced vibrations case, the single mode approach (SMA) is used the get a thirddegree equation solved by the classical Cardan method, in which the frequency ratio and the excitation level are the inputs and the real solution corresponds to the normalised mode shape contribution coefficient, the complex solutions being simply ignored.

\section{General formulation}

\subsection{Determination of the linear mode shapes:}

Consider a uniform Euler-Bernoulli beam, with $\mathrm{N}$ flexible supports of stiffness $\mathrm{K} j$, subjected to transverse free vibrations and having the following geometrical and material characteristics: (L: length, E: Young's modulus, S: Cross-sectional area, I: second moment of inertia of cross section, $r$ : radius of gyration, $\rho$ : mass per unit length). To be able and examine all possible beam end conditions, translational springs $k t_{1}, k t_{2}$, and rotational springs $k \theta_{3}, k \theta_{4}$, are used as shown in Fig. 1 .

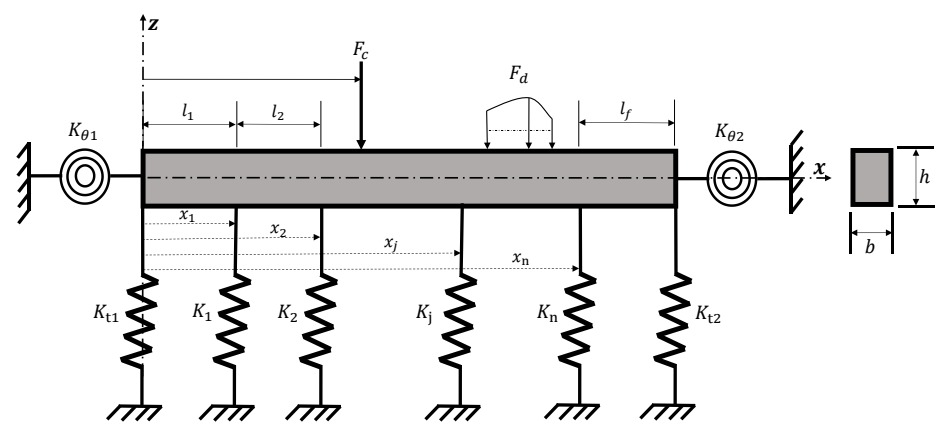

Fig. 1. Physical model of a Multiplan beam resting on arbitrary flexible supports

The general solution of the beam transverse vibration equation in the $\mathrm{j}^{\text {th }}$ span is given by:

$$
\begin{gathered}
\mathbf{w}_{j i}(\eta)=\mathbf{a}_{j} \cosh \left(\boldsymbol{\beta}_{i} L\left(\eta-\eta_{j-1}\right)\right)+\mathbf{b}_{j} \sinh \left(\boldsymbol{\beta}_{i} L\left(\eta-\eta_{j-1}\right)\right)+\mathbf{c}_{j} \cos \left(\boldsymbol{\beta}_{i} L\left(\eta-\eta_{j-1}\right)\right)+\mathbf{d}_{j} \sin \left(\boldsymbol{\beta}_{i} L\left(\eta-\eta_{j-1}\right)\right) \\
\eta_{j-1} \leq \eta \leq \eta_{j} ; \text { for }: j=1,2, \ldots, \mathrm{n}
\end{gathered}
$$

Where: $\beta_{i} L=L \sqrt[4]{\frac{\rho S \omega_{i}^{2}}{E I}}$ for: $i=1,2, .$. are the dimensionless frequencies, obtained numerically by the Newton-Raphson algorithm, whereas the other parameters $\mathbf{a}_{j}, \mathbf{b}_{j}, \mathbf{c}_{j}, \mathbf{d}_{j}$ are computed by means of the following conditions: 
The end conditions (at the left end) [5]:

$$
\left.\frac{\mathrm{d}^{3} \mathbf{w}_{1 i}(\eta)}{\mathrm{d} \eta^{3}}\right|_{\eta 0}=-\left.\mathrm{K}_{t 1} \mathbf{w}_{1 i}(\eta)\right|_{\eta 0} ;\left.\frac{\mathrm{d}^{2} \mathbf{w}_{1 i}(\eta)}{\mathrm{d} \eta^{2}}\right|_{\eta 0}=\left.\mathrm{K}_{\theta 1} \frac{\mathrm{d} \mathbf{w}_{1 i}(\eta)}{\mathrm{d} \eta}\right|_{\eta 0}
$$

The end conditions (at the right end):

$$
\left.\frac{\mathrm{d}^{3} \mathbf{w}_{(n+1) i}(\eta)}{\mathrm{d} \eta^{3}}\right|_{\eta 1}=\left.\mathrm{K}_{\mathrm{t} 2} \mathbf{w}_{(n+1) i}(\eta)\right|_{\eta l} ;\left.\frac{\mathrm{d}^{2} \mathbf{w}_{(n+l) i}(\eta)}{\mathrm{d} \eta^{2}}\right|_{\eta 1}=-\left.\mathrm{K}_{\theta 2} \frac{\mathrm{d} \mathbf{w}_{(n+l) i}(\eta)}{\mathrm{d} \eta}\right|_{\eta l}
$$

The compatibility conditions are given by [6]:

$$
\begin{array}{r}
\left.\mathbf{w}_{j i}(\eta)\right|_{\eta j}=\left.\mathbf{w}_{(j+1) i}(\eta)\right|_{\eta j}=0 ;\left.\frac{\mathrm{d} \mathbf{w}_{j i}(\eta)}{\mathrm{d} \eta}\right|_{\eta j}=\left.\frac{\mathrm{d} \mathbf{w}_{(j+1) i}(\eta)}{\mathrm{d} \eta}\right|_{\eta j} \\
\left.\frac{\mathrm{d}^{2} \mathbf{w}_{j i}(\eta)}{\mathrm{d} \eta^{2}}\right|_{\eta \mathrm{j}}=\left.\frac{\mathrm{d}^{2} \mathbf{w}_{(j+1) i}(\eta)}{\mathrm{d} \eta^{2}}\right|_{\eta \mathrm{j}} ;\left.\frac{\mathrm{d}^{3} \mathbf{w}_{j i}(\eta)}{\mathrm{d} \eta^{3}}\right|_{\eta \mathrm{j}}=\left.\frac{\mathrm{d}^{3} \mathbf{w}_{(j+l) i}(\eta)}{\mathrm{d} \eta^{3}}\right|_{\eta \mathrm{j}}+\mathbf{K}_{j} \mathbf{w}_{j i}\left(\eta_{j}\right) /(\mathrm{EI})
\end{array}
$$

The relationship between the $(j+1)^{\text {th }}$ and $j^{\text {th }}$ span's coefficients is given by eqs.(6-9), leading to the following equation, in which $\mathbf{T}_{j(4 \times 4)}$ is the transfer matrix corresponding to the $j^{\text {th }}$ spring:

$$
\left\{\begin{array}{c}
a_{j+1} \\
b_{j+1} \\
c_{j+1} \\
d_{j+1}
\end{array}\right\}=\mathbf{T}_{j}\left\{\begin{array}{c}
a_{j} \\
b_{j} \\
c_{j} \\
d_{j}
\end{array}\right\} \text { This leads to a system of matrix series: }\left\{\begin{array}{c}
a_{j+1} \\
b_{j+1} \\
c_{j+1} \\
d_{j+1}
\end{array}\right\}=\prod_{i=j}^{1} \mathbf{T}_{i}\left\{\begin{array}{c}
a_{1} \\
b_{1} \\
c_{1} \\
d_{1}
\end{array}\right\}
$$

Eq.(1-11) leads to the homogeneous system:

$$
\left[\begin{array}{c}
\mathbf{B}_{2 x 4} \cdot \prod_{N}^{1} \mathbf{T}_{i} \\
\mathbf{A}_{2 x 4}
\end{array}\right]\left\{\begin{array}{l}
a_{1} \\
b_{1} \\
c_{1} \\
d_{1}
\end{array}\right\}=\mathbf{M}\left\{\begin{array}{l}
a_{1} \\
b_{1} \\
c_{1} \\
d_{1}
\end{array}\right\}=0
$$

Where $\mathbf{B}_{\mathbf{2} \times 4}$ and $\mathbf{A}_{\mathbf{2} \times 4}$ are the matrix featuring the right and the left boundary conditions respectively. Which may have non-trivial solutions only if: $\operatorname{det}\left(\mathbf{M}_{4 \times 4}\right)=0$

In which $\mathbf{B}_{2 \times 4}$ and $\mathbf{A}_{4 \times 4}$ are the matrices corresponding to the right and the left end conditions. The $\beta_{i} \mathrm{~L}$ are the unknowns to find by Newton-Raphson algorithm [7], and the constants $b_{1}, c_{1}$, $d_{1}$, are calculated as functions of $a_{1}$, set to unity. Subsequently, the mode shapes, their $1^{\text {st }}$, $2^{\text {nd }}$, and $3^{\text {rd }}$ derivatives are computed numerically. In addition, numerical integration is performed in order to calculate the parameters used in the nonlinear analysis.

\subsection{Nonlinear formulation}

The multi-span beam total strain energy can be written as [2]:

$$
\mathbf{V}=\mathbf{V}_{\text {Nlin }}+\mathbf{V}_{\text {lin }}+\mathbf{V}_{\text {springs }}
$$


Where: $\mathbf{V}_{\text {Nin }}=\frac{E S}{8 L}\left[\int_{0}^{L}\left(\frac{\partial \mathbf{w}(x, t)}{\partial x}\right)^{2} d x\right]^{2} ; \mathbf{V}_{\text {lin }}=\frac{E I}{2} \int_{0}^{L}\left(\frac{\partial^{2} \mathbf{w}(x, t)}{\partial x^{2}}\right)^{2} d x ; \mathbf{V}_{\text {springs }}=\frac{1}{2} \sum_{j=1}^{N} k_{j} \mathbf{w}\left(x_{j}, t\right)^{2}$

The beam kinetic energy is given by: $\quad \mathbf{T}=\frac{1}{2} \rho S \int_{0}^{L}\left(\frac{\partial \mathbf{w}(x, t)}{\partial x}\right)^{2} d x$

Using a generalized parametrization and the usual summation convention, the transverse displacement can be written as:

$$
\mathbf{w}(x, t)=\mathbf{q}_{i}(t) \mathbf{w}_{i}(x)=\mathbf{a}_{i} \mathbf{w}_{i(x)} \sin (\omega t)
$$

The energy expressions are discretised as follows:

$$
\mathbf{T}=\frac{1}{2} \omega^{2} \mathbf{a}_{\mathbf{i}} \mathbf{a}_{\mathbf{j}} \cos ^{2}(\omega t) \mathbf{m}_{\mathrm{ij}} ; \mathbf{V}_{\text {Lin }}+\mathbf{V}_{\text {springs }}=\frac{1}{2} \mathbf{a}_{\mathbf{i}} \mathbf{a}_{\mathbf{j}} \mathbf{k}_{\mathbf{i j}} \sin ^{2}(\omega t) ; \mathbf{V}_{\text {Nin }}=\frac{1}{2} \mathbf{a}_{\mathbf{i}} \mathbf{a}_{\mathbf{j}} \mathbf{a}_{\mathbf{k}} \mathbf{a}_{\mathbf{l}} \mathbf{b}_{\mathbf{i j k}} \sin ^{4}(\omega t)
$$

Where $b_{\mathrm{ijk}}$ represents the nonlinearity tensor, $\mathbf{k}_{i j}$ denotes the rigidity matrix, and $\mathbf{m}_{i j}$ stands for the mass matrix. The amplitude $\mathbf{a}_{i}$ are unknown as well as the frequency $\omega$.

The dynamic behaviour of the structure may be obtained by Hamilton's principle for a conservative system [8], which leads to:

$$
\delta \int_{0}^{2 \pi / \omega}(\mathbf{V}-\mathbf{T}+\mathbf{F}) \mathrm{d} t=0
$$

Using Lagrange's equations, based on expressions (8) to (11), and then the HBM leads to:

$$
2 \mathbf{a}_{i} \mathbf{k}_{i r}+3 \mathbf{a}_{i} \mathbf{a}_{j} \mathbf{a}_{k} \mathbf{b}_{i j k r}-2 \omega^{2} a_{i} \mathbf{m}_{i r}=\mathbf{f}_{i} ; \text { with }: \mathrm{r}=1 \ldots \mathrm{N}
$$

In which $\boldsymbol{f}_{\mathrm{i}}$, is the generalised forces associated to the external forces applied to the $i^{\text {th }}$ mode. In order to get dimensionless parameters, the following parameterization are taken:

$$
\begin{aligned}
& w^{*}(\eta)=\frac{\mathbf{w}\left(\frac{x}{L}\right)}{r} ; \frac{\mathbf{k}_{i j}}{\mathbf{k}_{i j}^{*}}=\frac{E I r^{2}}{L^{3}} ; \frac{\omega^{2}}{\omega^{* 2}}=\frac{E I}{\rho S L^{4}} ; \frac{\mathbf{m}_{i j}}{\mathbf{m}_{i j}{ }^{*}}=\rho S r^{2} L ; \frac{\mathbf{b}_{i j k l}}{\mathbf{b}^{*}{ }_{i j k l}}=\frac{E I r^{2}}{L^{3}} ; \mathbf{f}_{\mathbf{i}}^{*}=\frac{f L^{4}}{E I r} \int_{0}^{1} \mathbf{w}(\eta) d \eta \\
& \mathbf{k}_{i j}^{*}=E I \int_{0}^{1} \frac{\partial^{2} \mathbf{w}_{i}^{*}}{\partial \eta^{*}} \frac{\partial^{2} \mathbf{w}_{j}}{\partial \eta^{2}} d \eta+\sum_{t=1}^{N} k_{t}^{*} \mathbf{w}_{i}^{*}\left(x_{t}\right) \mathbf{w}_{j}^{*}\left(x_{t}\right) ; \mathbf{b}_{i j k l}^{*}=\alpha \int_{0}^{1} \frac{\partial \mathbf{w}_{\mathbf{i}}^{*}}{\partial \eta} \frac{\partial \mathbf{w}_{\mathbf{j}}^{*}}{\partial \eta} d \eta \int_{0}^{1} \frac{\partial \mathbf{w}_{\mathbf{k}}^{*}}{\partial \eta} \frac{\partial \mathbf{w}^{*}}{\partial \eta} d \eta
\end{aligned}
$$

Eq. (19) becomes: $\quad 2 \mathbf{a}_{i} \mathbf{k}_{i r}^{*}+3 \mathbf{a}_{i} \mathbf{a}_{j} \mathbf{a}_{k} \mathbf{b}_{i j k r}{ }^{*}-2 \omega^{* 2} \mathbf{a}_{i} \mathbf{m}_{i r}{ }_{i r}=\mathbf{f}_{i}^{*} ;$ avec $: r=1 \ldots N$

In single mode approach [1,9], only one mode is assumed to contribute to the nonlinear

response Thus, eq. (22) becomes: $\left(\frac{\omega^{*}}{\omega^{*}}\right)^{2}=1+\frac{3}{2}\left(\frac{b_{1111}{ }^{*}}{k^{*}{ }_{11}}\right) a_{1}{ }^{2}-\frac{\mathbf{f}_{i}{ }^{*}}{\mathbf{k}_{i i}{ }^{*} a_{1}} ; \omega_{L}{ }^{* 2}=\frac{k_{11}{ }^{*}}{m_{11}{ }^{*}}$

This equation is solved by Cardan method where $\left(\frac{\omega^{*}}{\omega_{L}^{*}}\right)$ is given and the deflection amplitudes $a_{1}$ are found and plotted in a way to select just the real solutions and to ignore the complex ones. The generalised dimensionless forces $\mathbf{f}^{{ }^{*} \mathrm{c}}$ and $\mathbf{f}^{\boldsymbol{*}_{\mathrm{d}}}$ corresponding respectively 
to a concentrated $\mathbf{F}^{\mathbf{c}}$, located at $\eta_{0}$, and a uniformly distributed force $\mathbf{F}^{\mathbf{d}}$ are respectively given by:

$$
\mathbf{f}^{* \mathbf{c}}=\frac{\mathbf{F}^{\mathfrak{c}} L^{3}}{E I r} \mathbf{w}^{*}\left(\eta_{0}\right) ; \mathbf{f}^{* \mathbf{d}}=\frac{\mathbf{F}^{\mathbf{d}} L^{4}}{E I r} \int_{0}^{1} \mathbf{w}^{*}(\eta) d \eta
$$

\section{Numerical results and discussion}

In this section, the case of a clamped-clamped beam with five springs is studied. The springs are positioned uniformly $\left(\eta_{1}=1 / 6, \eta_{2}=2 / 6, \eta_{3}=3 / 6, \eta_{4}=4 / 6, \eta_{5}=5 / 6\right)$. The dimensionless stiffness is taken equal to $\mathrm{k}_{1}=1000, \mathrm{~K}_{2}=3000, \mathrm{k}_{3}=5000, \mathrm{k}_{4}=3000, \mathrm{k}_{5}=1000$.
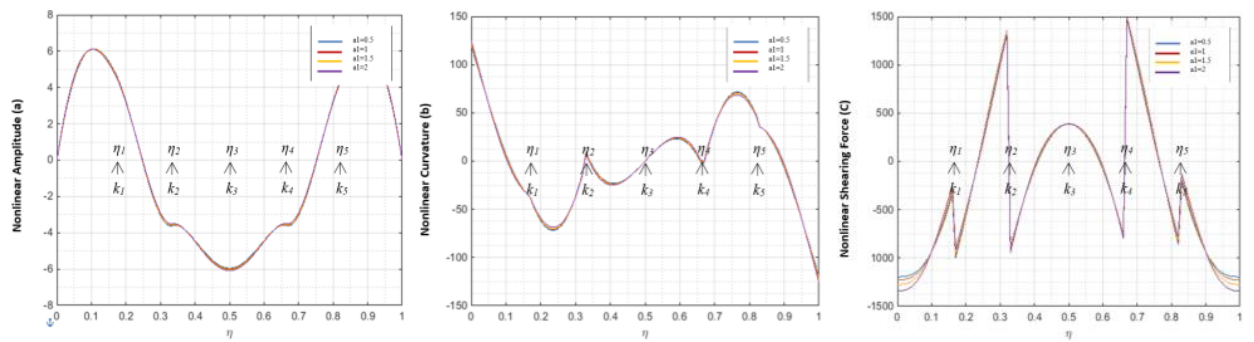

Fig. 2. First Mode, Non-linear curvature and Non-linear shearing force of B5IFS uniformly distributed for various vibrations level, in the free vibration case.

The normalized nonlinear mode shapes, curvature and shearing force are respectively plotted in (Fig. 2. a, b, c); for various vibration amplitudes, the effect of geometric non-linearity has been clearly observed.
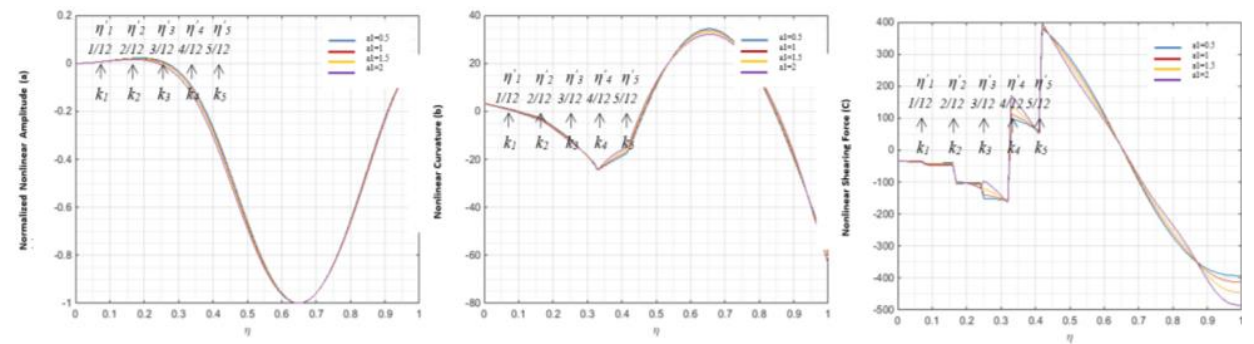

Fig. 3. First Mode, Non-linear curvature and Non-linear shearing force of B5IFS concentrated on the left middle spaced with $l_{j}=1 / 12$, for various vibrations level.
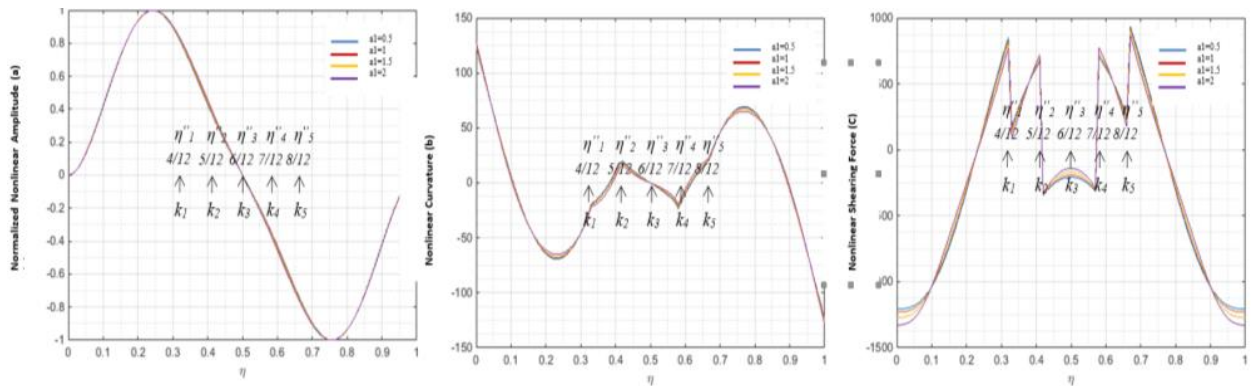

Fig. 4. First Mode, Non-linear curvature and Non-linear shearing force of B5IFS concentrated on the centre spaced with $l_{\mathrm{j}}=1 / 12$, for various vibrations level. 

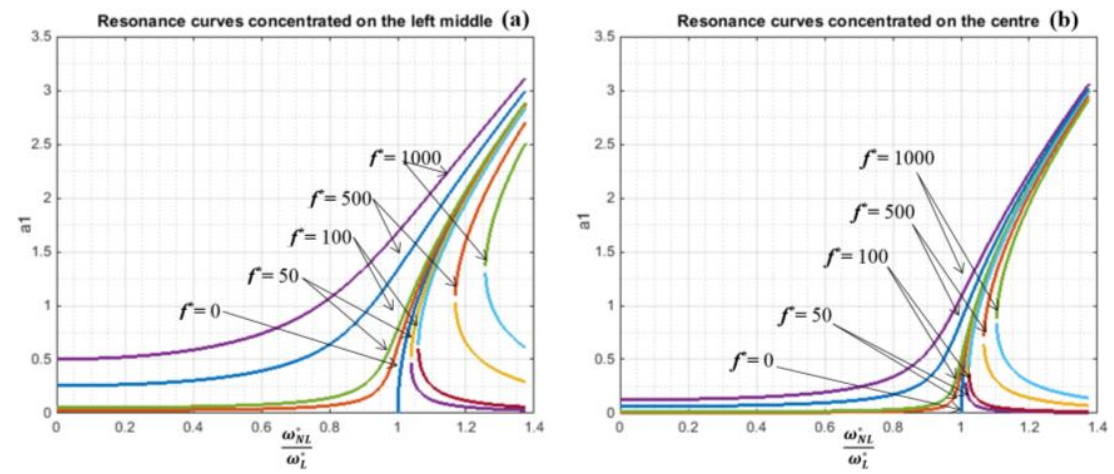

Fig. 5. Nonlinear frequency response functions, based on the single mode approach B5IFS concentrated on the left middle and on the centre respectively

By assuming a single mode approach used previously in [1], the Eq. (23) is represented in (Fig. 5.) in the neighbourhood of the fundamental mode shape for various values of the harmonic dimensionless excitation amplitude $f_{1}^{*}$ for the case of a B5IFS concentrated on the left middle, and on the centre respectively. The springs position effect on the dynamic behaviour of the forced structure is clearly observed between the two cases.

\section{Conclusion}

The nonlinear forced vibrations of a BNIFS with intermediate springs positioned at arbitrary locations, supported by translational and rotational springs at the two ends, have been examined analytically. The dynamic equation was developed at each intervals of the beam span with appropriate end and compatibility conditions. The different tensors were computed using SIMPSON method and the Newton-Raphson iterations algorithm has been used to solve the equations, to obtain the mode shapes, slopes, curvatures and shearing forces. The non nonlinear effects has been clearly shown for various excitation levels, whereas the resonance curves are obtained by using SMA which gives a better approximation of $4 \%$ according to [1-4].

\section{References}

[1] L. Azrar, R. Benamar, and R. G. White, J. Sound Vib., 224, no. 2, pp. 183-207, (1999).

[2] R. Benamar, M. Bennouna, R. G. White, J. Sound Vib., 149, 179-195(1991).

[3] R. Benamar. Ph.D thesis. (University of Southampton. 1990).

[4] M. Bennouna, R. G. White, J. Sound Vib., 96, 309-331 (1984).

[5] A. Ahmed and B. Rhali, Int. J. Innov. Res. Sci. Eng. Technol., 6, no. 6, pp. 11036-11042, (2017).

[6] Igor A. Karnovskiı̌ and O. I. Lebed, Formulas for structural dynamics : tables, graphs, and solutions. (2001).

[7] J. Kiusalaas, Numerical Methods in Engineering with MATLAB, Second Edi. (2010).

[8] M. Géradin and D. J. Rixen, Mechanical Vibrations: Theory and Application to Structural Dynamics. (2015).

[9] M. El Kadiri, R. Benamar, and R. G. White, J. Sound Vib., 249, no. 2, pp. 263-305, (2002). 